\title{
Beneficial Effects of Appropriate Sleep Duration on Depressive Symptoms and Perceived Stress Severity in a Healthy Population in Korea
}

\author{
Hyo-Min Kim', Sang Won Lee ${ }^{2, *}$ \\ 'Department of Family Medicine, Kyungpook National University Medical Center, Daegu, Korea \\ ${ }^{2}$ Department of Psychiatry, Kyungpook National University School of Medicine, Daegu, Korea
}

\begin{abstract}
Background: Recently, several institutions, including the American Academy of Sleep Medicine, the Sleep Research Society, and the National Sleep Foundation, have made consensus recommendations concerning appropriate sleep duration for adults. Although numerous studies conducted in Western populations have provided evidence of the harmful effects of short or long sleep duration on mental health, it is still unclear whether these consensus recommendations are appropriate in Korean culture.

Methods: Data from 1,892 subjects with no history of medical or psychiatric diagnoses were selected from the Korea National Health and Nutrition Examination Survey of 2014. Subjects were divided into seven groups based on their sleep duration $(\leq 4,5,6,7,8,9$, and $\geq 10$ hours). Depressive symptoms were measured using the Patient Health Questionnaire-9 and perceived stress severity was evaluated using a Likert-type scale. Group differences in depressive symptoms and severity of stress were analyzed using an analysis of covariance.

Results: Depressive symptoms in subjects with sleep duration of $\leq 4$ hours $(5.7 \pm 5.9)$ or 5 hours $(3.4 \pm 3.9)$ were higher than in subjects with a sleep duration of $7(2.2 \pm 2.9)$ or 8 hours $(2.2 \pm 2.9)$ (corrected $\mathrm{P}<0.05)$. Furthermore, subjects with a short sleep duration ( 5 hours or below) had greater perceived stress severity than subjects with a sleep duration of 7 or 8 hours (corrected $\mathrm{P}<0.05$ ).

Conclusion: Our results suggest that maintaining an appropriate sleep duration as found in the recent consensus recommendation is important for mental health, even in healthy subjects without any medical or psychiatric illnesses, in Korea.
\end{abstract}

Keywords: Sleep; Depression; Stress; Healthy Lifestyle

Received: August 9, 2016, Revised: November 16, 2016, Accepted: December 1, 2016

${ }^{*}$ Corresponding Author: Sang Won Lee https://orcid.org/0000-0002-3537-7110

Tel: +82-53-420-6514, Fax: +82-53-426-5361, E-mail: leesangwon.psy@gmail.com 


\section{INTRODUCTION}

Verifying appropriate sleep duration is important for medical and psychiatric health and many researchers have tried to provide guidelines for healthy sleep duration. In 2015, the American Academy of Sleep Medicine and the Sleep Research Society suggested 7 or more hours per night on a regular basis to promote optimal health in adults (18 to 60 years), ${ }^{1)}$ and the National Sleep Foundation recommended a sleep range of 7 to 9 hours per day for young adults (18 to 24 years) and adults (25 to 64 years). ${ }^{2)}$

The association between sleep duration and mental health has been addressed in previous studies. Short sleep duration has been linked to various psychiatric disorders, including depressive disorder, ${ }^{3,4)}$ depression-related suicidal behavior, ${ }^{5)}$ and anxiety disorder, ${ }^{3)}$ In addition, a recent meta-analysis of prospective studies showed that both short and long sleep duration were significantly related to an increased risk of depression. ${ }^{6}$ However, insomnia or hypersomnia is one of the common symptoms in depressive disorder and the presence of depression is a significant factor in short or long sleep duration. ${ }^{7)}$ Furthermore, various medical diseases and disease-related medications can cause insomnia or hypersomnia. It is important to consider these confounders when analyzing the relationship between sleep duration and mental health, with previous well-designed studies controlling for these confounding factors. ${ }^{8-10)}$ The Korea National Health and Nutrition Examination Survey (KNHANES) was conducted from 1998 to 2014 in Korea. This survey collected information regarding health and nutritional status, including individual histories of the diagnosis of most medical diseases and depression. Therefore, the relationship between sleep duration and mental health can be appropriately measured using data from the KNHANES.

In addition, the social environment can affect sleep behaviors. Most studies have reported the relationship between sleep duration and depression were conducted in Western culture. ${ }^{6)}$ Among the Organization for Economic Cooperation and Development (OECD) states, mean sleep duration for Koreans was the shortest (469 minutes), 33 minutes shorter than the OECD average. ${ }^{11)}$ Differences in social environment may affect the relationship between sleep duration and mental health. Therefore, consensus recommendations need to be verified in Korean culture. It is important to identify the appropriate sleep duration to promote mental health in Korean adults.

The present study aimed to investigate the cross-sectional association between sleep duration and depressive symptoms, depressionrelated quality of life, and daily stress in a healthy population in Korea.

\section{METHODS}

\section{Study Population}

We used the publicly available data from the KNHANES in 2014. The individual histories of the diagnosis of most medical diseases, including cancer, hypertension, and depression, were evaluated in the KNHANES. Subjects were asked whether they had ever been diag- nosed by a doctor with any of following conditions: hypertension, dyslipidemia, stroke, ischemic heart disease, arthritis, tuberculosis, asthma, diabetes, thyroid disease, cancer, atopic dermatitis, allergic rhinitis, chronic liver disease, chronic kidney disease, and depression. We excluded the data of all subjects with any of these diseases. We selected the data from the KNHANES 2014 because a self-reported questionnaire that quantitatively measures depressive symptoms was included. Of the total 7,550 subjects, there were 4,423 subjects aged between 20 and 65 years. Finally, 1,892 subjects without a history of medical or psychiatric (depression) diagnosis were selected for our study. The KNHANES 2014 was approved by the institutional review board of the Korea Centers for Disease Control and Prevention (2013-12EXP-03-5C). Additionally, this study was approved by the institutional review board of the Kyungpook National University Medical Center (KNUMC 2016-09-035).

\section{Sleep Duration and Other Variables}

Sleep duration was evaluated using the question "On average, how much do you sleep each day?" Subjects were classified into seven groups based on their sleep duration: $\leq 4,5,6,7,8,9$, and $\geq 10$ hours. Depressive symptoms were evaluated using the Patient Health Questionnaire-9 (PHQ-9). ${ }^{12)}$ The Korean version of the PHQ-9 has been shown to have good reliability and validity. ${ }^{13)}$ Perceived stress severity was measured using the question "How much do you feel stress in daily life?" Likert-type scales were used to evaluate both perceived stress severity (1: extremely, 2: much, 3: slightly, and 4: scarcely) and quality of life regarding anxiety/depression, which is a part of the EQ$5 \mathrm{D}$ and developed by the EuroQol group, ${ }^{14)}$ (1: none, 2: moderate, and 3: severe). In addition, the proportional size of the high stress group was evaluated using the number of subjects who rated extreme or much stress in the perceived stress severity questionnaire. Basic epidemiological characteristics, such as age, gender, education, and household income, were also included. Physical activities were elicited using the following question: How many days did you perform physical activities like walking (or strengthening or stretching) during the past week?

\section{Statistical Analysis}

An analysis of variance (ANOVA) for continuous variables and the chisquare test for categorical variables were used to measure group differences among the seven sleep duration groups. Post hoc analysis between groups was applied using the Bonferroni test for multiple comparisons. In the post hoc analysis of the chi-square test, an adjusted residual method was used to measure the statistical significance (P-value of $0.05 / 14$ correcting for multiple comparisons). ${ }^{15)}$ An additional analysis of covariance (ANCOVA) was performed to control for the effects of age, sex, level of education, and household income. All analyses were performed using PASW SPSS ver. 18.0 (SPSS Inc., Chicago, IL, USA), and a P-value $<0.05$ was considered statistically significant. 


\section{RESULTS}

The mean sleep duration of our 1,892 subjects was $6.9 \pm 1.3$ hours. Overall, our healthy subjects demonstrated very low scores of depressive symptoms and slight stress (PHQ-9, 2.5 \pm 3.3 ; perceived stress severity, 2.8 \pm 0.7 ; and EQ-anxiety/depression, 1.1 \pm 0.3 ). The shortest sleep duration group tended to be older, had fewer years of education, and had a lower household income (corrected $\mathrm{P}<0.05$ ). In the ANOVA and following post hoc analysis, the subjects with a short sleep duration (5 hours or below) had more depressive symptoms, higher perceived stress severity, and lower quality of life related to anxiety/depression than those with a sleep duration of 7 or 8 hours (corrected $\mathrm{P}<0.05$ ) (Table 1). Subjects with a long sleep duration (10 hours or above) did not show any significant differences compared with those who had a 7-, 8-, or 9-hour sleep duration. There were also no significant group differences in weekly physical activities. In the ANCOVA analysis controlling for the effects of age, sex, level of education, and household income, the overall group differences in PHQ-9 ( $F=15.03$, $\mathrm{P}<0.001)$, EQ-anxiety/depression $(\mathrm{F}=5.59, \mathrm{P}<0.001)$, and perceived stress severity $(\mathrm{F}=8.57, \mathrm{P}<0.001)$ were maintained (Table 2). Furthermore, the relationship between sleep duration and the measurements of the PHQ-9, EQ-anxiety/depression, and perceived stress severity showed similar patterns of U-shaped or inverted-U-shaped graphs (Figure 1).

\section{DISCUSSION}

This study provides evidence that short sleep duration (5 hours or below) can have negative effects on depressive symptoms, perceived stress severity, and quality of life related to anxiety/depression. Our results suggest that 7 or 8 hours sleep per night is sufficient to cope with daily stress and/or emotional burdens.

Subjects with the shortest sleep duration (4 hours or below) were likely to be older and have lower levels of income and education. A previous study in Korea also found a relationship between lower socioeconomic status and short sleep duration. ${ }^{7)}$ We performed an ANCOVA analysis to mitigate the effects of the differences in basic characteristics and found results similar to those of the ANOVA analysis.

Though the overall level of depressive symptoms and perceived stress were low in our healthy subjects, short sleep duration (5 hours or below) was associated with a higher level of depressive symptoms and perceived stress severity. Short sleep duration can be a risk factor for psychiatric disorders including major depressive disorder and posttraumatic stress disorder after a severe stressful event. ${ }^{16)}$ Our results suggest that short sleep duration may be related to a failure in processing common daily stresses or emotional stimuli in a healthy way. Short sleep duration has been shown to predict persistent psychological distress in the near future in a prospective study with young adults ${ }^{17)}$ and showed a relationship with psychological distress in a cross-sectional

Table 1. Group differences in epidemiological and psychological characteristics among groups with different sleep duration

\begin{tabular}{|c|c|c|c|c|c|c|c|c|c|}
\hline \multirow[b]{2}{*}{ Variable } & \multicolumn{7}{|c|}{ Sleep duration } & \multicolumn{2}{|r|}{ Statistics } \\
\hline & $\leq 4(n=58)$ & $5(n=163)$ & $6(n=503)$ & $7(n=593)$ & $8(n=444)$ & $9(n=66)$ & $\geq 10(n=65)$ & P-value & $\begin{array}{c}\text { Post-hoc test } \\
\text { (Bonferroni correction) }\end{array}$ \\
\hline Age (y) & $45.7 \pm 12.2$ & $42.7 \pm 11.2$ & $42.0 \pm 10.8$ & $41.3 \pm 11.4$ & $40.7 \pm 11.9$ & $35.3 \pm 12.6$ & $39.9 \pm 14.2$ & $<0.001^{\natural}$ & $9>4,5,6,7,8 \& 8>4$ \\
\hline Male sex & $26(44.8)$ & $74(45.4)$ & 247 (49.1) & $267(45.0)$ & $182(41.0)$ & 17 (25.8) & $29(44.6)$ & $0.014^{\star *}$ & $4,5,6,7,8,10>9$ \\
\hline Education ( $\leq 12$ y) & $37(63.8)$ & $80(49.1)$ & $229(45.5)$ & 234 (39.5) & $192(43.2)$ & $20(30.3)$ & $34(52.3)$ & $0.001^{\star *}$ & $5,6,7,8,9,10>4$ \\
\hline Household income $(\leq 250)^{\star}$ & $29(50.9)^{\dagger}$ & $56(34.6)^{\dagger}$ & $141(28.1)$ & $163(27.5)$ & $131(29.5)$ & $26(39.4)$ & $35(53.8)$ & $<0.001^{\star \star}$ & $4,10>5,6,7,8,9$ \\
\hline Patient Health Questionnaire-9 & $5.7 \pm 5.9$ & $3.4 \pm 3.9^{\dagger}$ & $2.3 \pm 3.0^{\dagger}$ & $2.2 \pm 2.9^{\ddagger}$ & $2.2 \pm 2.9^{\dagger}$ & $2.6 \pm 2.8^{\dagger}$ & $3.0 \pm 4.6^{\dagger}$ & $<0.001^{\uparrow}$ & $4>5,6,7,8,9,10 \& 5>6,7,8$ \\
\hline Perceived stress severity & $2.5 \pm 0.9$ & $2.7 \pm 0.7$ & $2.8 \pm 0.7$ & $2.9 \pm 0.7$ & $2.9 \pm 0.7$ & $3.0 \pm 0.7$ & $2.8 \pm 0.8^{\S}$ & $<0.001^{\Uparrow}$ & $7,8,9>4,5 \& 7>6$ \\
\hline Percentage of high stress & $25(43.1)$ & $60(36.8)$ & $147(29.0)$ & $131(22.1)$ & $97(21.8)$ & $17(25.8)$ & $15(27.8)^{\S}$ & $<0.001^{\star \star}$ & $4,5>6,7,8,9,10$ \\
\hline EuroQol-anxiety/depression & $1.21 \pm 0.45$ & $1.13 \pm 0.34$ & $1.08 \pm 0.28$ & $1.04 \pm 0.21$ & $1.05 \pm 0.24$ & $1.12 \pm 0.33$ & $1.09 \pm 0.29$ & $<0.001^{\Uparrow}$ & $4>6,7,8 \& 5>7,8$ \\
\hline \multicolumn{10}{|l|}{ Physical activity" } \\
\hline Walking & $4.4 \pm 2.7$ & $4.3 \pm 2.6$ & $3.9 \pm 2.6$ & $3.9 \pm 2.5$ & $3.8 \pm 2.6$ & $3.6 \pm 2.5$ & $3.5 \pm 2.5$ & $0.127^{\Uparrow}$ & \\
\hline Strengthening & $0.5 \pm 1.3$ & $0.7 \pm 1.4$ & $0.8 \pm 1.5$ & $0.7 \pm 1.4$ & $0.8 \pm 1.5$ & $0.7 \pm 1.4$ & $0.7 \pm 1.7$ & $0.904^{\Uparrow}$ & \\
\hline Stretching & $2.3 \pm 2.1$ & $2.0 \pm 2.0$ & $2.0 \pm 2.0$ & $1.9 \pm 2.0$ & $1.7 \pm 2.0$ & $2.0 \pm 2.1$ & $1.5 \pm 2.1$ & $0.073^{\Uparrow}$ & \\
\hline
\end{tabular}

Values are presented as mean \pm standard deviation or number (\%), unless otherwise specified.

*Unit is 10,000 Korean won/mo and a value of 250 was applied based on the mean of household income within the bottom $50 \%$ in 2014 . ${ }^{\dagger, \neq}$ sSeveral data were missing ( ${ }^{\dagger}$ one, ${ }^{\ddagger}$ two, and ${ }^{\S}$ eleven). "Unit is day/wk. "Analysis of variance or ${ }^{* \star}$ Chi-square test were used to measure group differences.

Table 2. Group differences in depressive symptoms and perceived stress severity after controlling for age, sex, education, and household income

\begin{tabular}{|c|c|c|c|c|c|c|}
\hline \multirow{2}{*}{ Variable } & \multicolumn{6}{|c|}{ Statistics } \\
\hline & Sum of squares & $\mathrm{df}^{*}$ & Mean square & $\mathrm{F}$ & P-value & Post-hoc test (Bonferroni correction) \\
\hline Patient Health Questionnaire-9 & 888.24 & 6 & 148.04 & 15.03 & $<0.001$ & $4>5,6,7,8,9,10 \& 5>6,7,8$ \\
\hline Perceived stress severity & 23.64 & 6 & 3.94 & 8.57 & $<0.001$ & $6>4 \& 7,8,9>4,5 \& 7>6$ \\
\hline EuroQol-anxiety/depression & 2.33 & 6 & 0.39 & 5.59 & $<0.001$ & $4,5>7,8$ \\
\hline
\end{tabular}

*Degrees of freedom. ${ }^{\dagger}$ Numbers used in post-hoc test represent sleep duration. Analysis of covariance was used to measure group differences. 
A
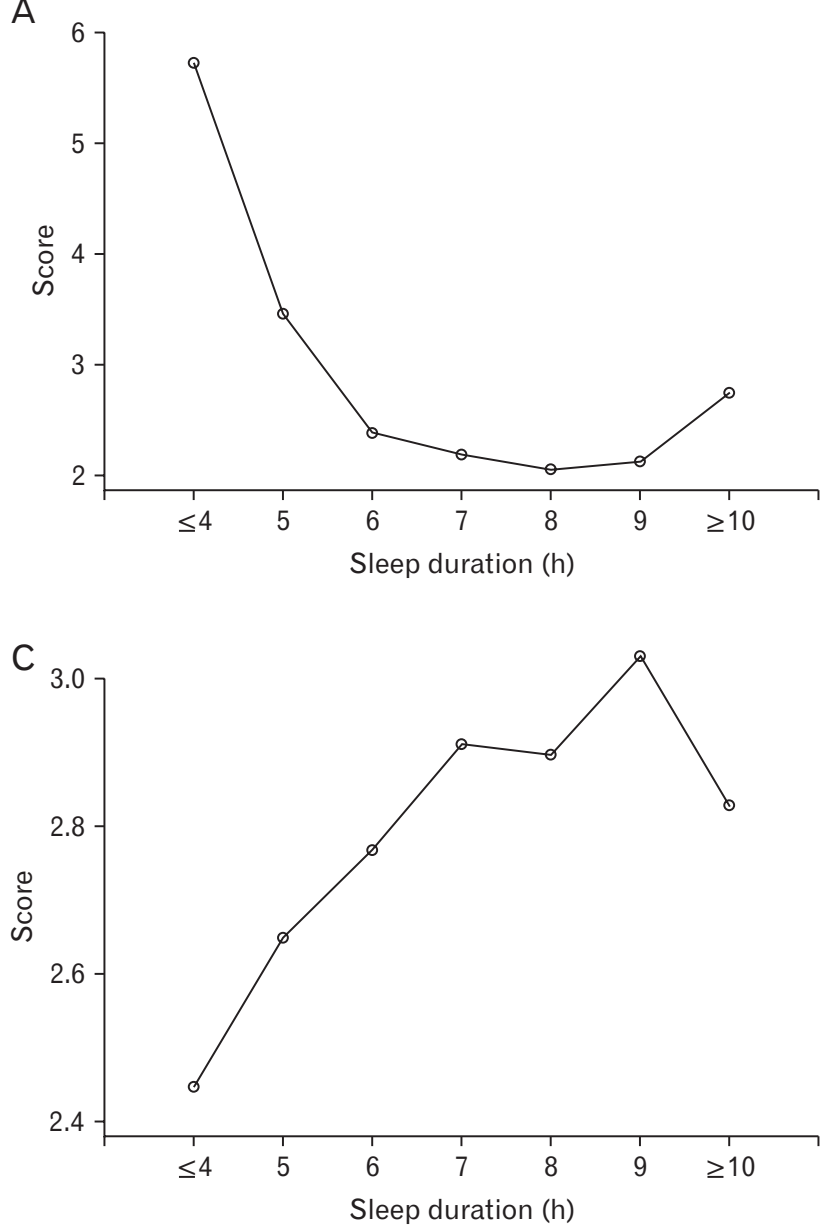

$\mathrm{B}$

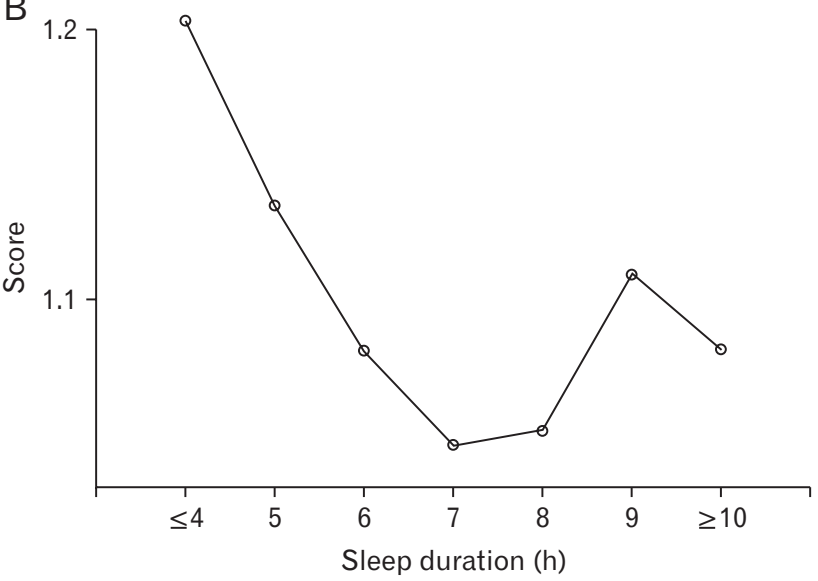

D

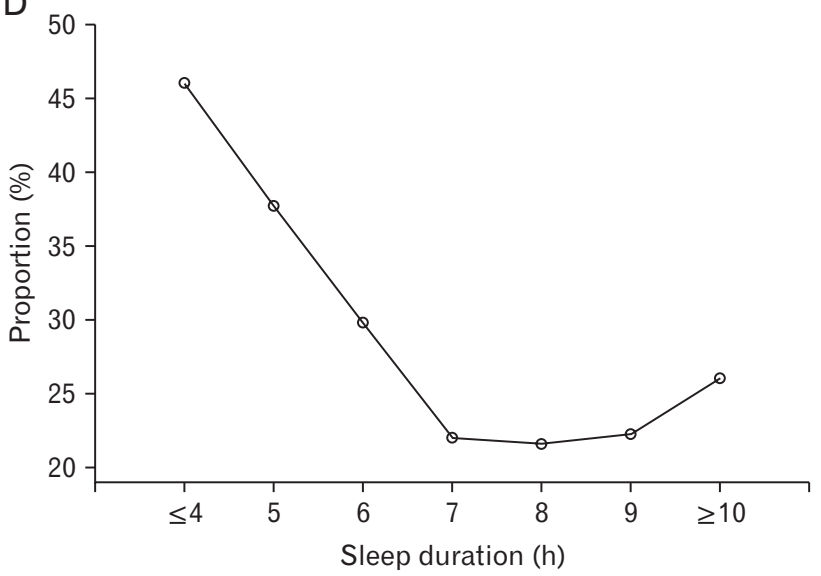

Figure 1. Graph of the psychological characteristics among sleep duration groups. (A) Patient Health Questionnaire-9. (B) EuroQol-anxiety/depression. (C) Perceived stress severity. (D) Proportion of high stress subjects. In the case of perceived stress severity (C), higher scores indicate a lower degree of stress severity.

and population-based study. ${ }^{18)}$ Furthermore, while significant effects of long sleep duration (10 hours or above) on depressive symptoms and perceived stress severity were not shown, the U- and inverted-Ushaped graphs (Figure 1) may indicate the possibility of harmful effects of long sleep duration. In sum, 7 or 8 hours (or possibly 9 hours) sleep duration showed favorable effects on subjects' overall mood and stress in a healthy population, and our results were in line with the consensus recommendation from several institutions.

In contrast to the results of the effects of sleep duration on mental health, we did not find any relationship between a short duration sleep and physical activities. A previous study showed that sufficient sleep can increase physical activity in children. ${ }^{19)}$ We did not observe overt effects of sleep duration on physical activities in the healthy subjects in our study. Recent studies showed no significant relationship between sleep duration and sedentary time ${ }^{20)}$ or physical activity. ${ }^{21)}$ Our results also suggest that short sleep duration may not disrupt daily activities, such as walking, strengthening, and stretching, in healthy subjects.

Some limitations of this study should be noted. First, our cross-sectional design cannot confirm a causal relationship between depressive symptoms and sleep duration. While subjects with medical diseases or depressive disorder were excluded in our study, subclinical depression or perceived stress can affect sleep duration. ${ }^{22)}$ Second, sleep duration was measured using a self-reported questionnaire, which can lead to recall bias. Third, while basic epidemiological characteristics were included as covariates in an ANCOVA analysis, the differences in epidemiological characteristics can be a confounding factor of our results.

In conclusion, our results suggest that an appropriate sleep duration of 7 or 8 hours (and possibly 9 hours) per night for adults, as stated in recent consensus recommendations, and maintaining a healthy sleep duration may be important for maintaining mental health, even in healthy subjects without any medical or psychiatric illnesses in Korea.

\section{CONFLICT OF INTEREST}

No potential conflict of interest relevant to this article was reported.

\section{REFERENCES}

1. Consensus Conference Panel, Watson NF, Badr MS, Belenky G, Bliwise 
DL, Buxton OM, et al. Joint consensus statement of the American Academy of Sleep Medicine and Sleep Research Society on the recommended amount of sleep for a healthy adult: methodology and discussion. Sleep 2015;38:1161-83.

2. Hirshkowitz M, Whiton K, Albert SM, Alessi C, Bruni O, DonCarlos L, et al. National Sleep Foundation's sleep time duration recommendations: methodology and results summary. Sleep Health 2015;1:40-3.

3. John U, Meyer C, Rumpf HJ, Hapke U. Relationships of psychiatric disorders with sleep duration in an adult general population sample. J Psychiatr Res 2005;39:577-83.

4. Kaneita Y, Ohida T, Uchiyama M, Takemura S, Kawahara K, Yokoyama E, et al. The relationship between depression and sleep disturbances: a Japanese nationwide general population survey. J Clin Psychiatry 2006;67:196-203.

5. Goodwin RD, Marusic A. Association between short sleep and suicidal ideation and suicide attempt among adults in the general population. Sleep 2008;31:1097-101.

6. Zhai L, Zhang H, Zhang D. Sleep duration and depression among adults: a meta-analysis of prospective studies. Depress Anxiety 2015;32:664-70.

7. Ryu SY, Kim KS, Han MA. Factors associated with sleep duration in Korean adults: results of a 2008 community health survey in Gwangju metropolitan city, Korea. J Korean Med Sci 2011;26:1124-31.

8. Paudel M, Taylor BC, Ancoli-Israel S, Blackwell T, Maglione JE, Stone $\mathrm{K}$, et al. Sleep disturbances and risk of depression in older men. Sleep 2013;36:1033-40.

9. Maglione JE, Ancoli-Israel S, Peters KW, Paudel ML, Yaffe K, Ensrud $\mathrm{KE}$, et al. Subjective and objective sleep disturbance and longitudinal risk of depression in a cohort of older women. Sleep 2014;37:1179-87.

10. Szklo-Coxe M, Young T, Peppard PE, Finn LA, Benca RM. Prospective associations of insomnia markers and symptoms with depression. Am J Epidemiol 2010;171:709-20.

11. Organization for Economic Cooperation and Development. Special focus: measuring leisure in OECD countries. In: Organization for Economic Cooperation and Development, editor. Society at a glance 2009: OECD social indicators. Paris: Organization for Economic Coopera- tion and Development; 2009. p. 19-50.

12. Kroenke K, Spitzer RL, Williams JB. The PHQ-9: validity of a brief depression severity measure. J Gen Intern Med 2001;16:606-13.

13. Park SJ, Choi HR, Choi JH, Kim KW, Hong JP. Reliability and validity of the Korean version of the patient health questionnaire-9 (PHQ-9). Anxiety Mood 2010;6:119-22.

14. EuroQol Group. EuroQol: a new facility for the measurement of health-related quality of life. Health Policy 1990;16:199-208.

15. Beasley TM, Schumacker RE. Multiple regression approach to analyzing contingency tables: post hoc and planned comparison procedures. J Exp Educ 1995;64:79-93.

16. Swinkels CM, Ulmer CS, Beckham JC, Buse N, Calhoun PS. The association of sleep duration, mental health, and health risk behaviors among U.S. Afghanistan/Iraq era veterans. Sleep 2013;36:1019-25.

17. Glozier N, Martiniuk A, Patton G, Ivers R, Li Q, Hickie I, et al. Short sleep duration in prevalent and persistent psychological distress in young adults: the DRIVE study. Sleep 2010;33:1139-45.

18. Cunningham TJ, Wheaton AG, Giles WH. The association between psychological distress and self-reported sleep duration in a population-based sample of women and men. Sleep Disord 2015;2015: 172064.

19. Khan MK, Chu YL, Kirk SF, Veugelers PJ. Are sleep duration and sleep quality associated with diet quality, physical activity, and body weight status?: a population-based study of Canadian children. Can J Public Health 2015;106:e277-82.

20. Saleh D, Janssen I. Interrelationships among sedentary time, sleep duration, and the metabolic syndrome in adults. BMC Public Health 2014;14:666.

21. Xiao Q, Arem H, Moore SC, Hollenbeck AR, Matthews CE. A large prospective investigation of sleep duration, weight change, and obesity in the NIH-AARP Diet and Health Study cohort. Am J Epidemiol 2013;178:1600-10.

22. Charles LE, Slaven JE, Mnatsakanova A, Ma C, Violanti JM, Fekedulegn $\mathrm{D}$, et al. Association of perceived stress with sleep duration and sleep quality in police officers. Int J Emerg Ment Health 2011;13:229-41. 